\title{
RESEARCH INTO INTELLIGENT TRANSPORT SYSTEMS (ITS) TECHNOLOGIES AND EFFICIENCY
}

\author{
Aldona Jarašūnienè \\ Transport Research Institute, Vilnius Gediminas Technical University, \\ Plytiness g. 27,LT-10105Vilnius, Lithuania.E-mail: ajarasuniene@yahoo.com
}

Received 27 November 2006; accepted 1 February 2007

\begin{abstract}
Intelligent Transport Systems work with information and control technologies which provide the core of ITS functions. Some of these technologies, like loop detectors, are well known to transportation professionals. However, there are a number of less familiar technologies and system concepts that are the key to ITS functions. The technical core of ITS is information and control technologies, but human factors are also vitally important, and potentially very complex. This paper introduces the main ITS enabling technologies and explains why transport professionals should involve human factor experts at an early stage of design of ITS equipment and facilities.
\end{abstract}

Keywords: Intelligent transport systems (ITS), information, control systems, research on ITS technologies, ITS efficiency.

\section{Introduction}

ITS purpose is to gather information about traffic conditions and traffic flows on roads and to present it in non-distorted form for control systems (GPS, route control and creating public transport control systems, commercial transport control systems, electronic payment and tax collecting systems, etc.) Control systems themselves can be defined by qualitative parameters: controlling scope, expedition, adaptation, controlling algorithm and collecting systems efficiencies, variety and utility of the information they deliver.

Intelligent electronic transportation control system usually contains such main parts: information collecting subsystem; information processing subsystem; information transmission subsystem; controlling subsystem; subsystem of interfaces between separate hierarchical levels.

Electronic ITS contain a set of technical tools connected to general information processing complex. Since at least two systems working according to common algorithms and connected using interfaces may be considered as minimal integrated system, in that way electronic ITS is also an integrated system [1] (video surveillance signal processing, controlling system, etc.). The main part of the system that collects information from video cameras consists of digital video cameras and special video signal processing and transmitting cards that are installed in proper road sections and crossroads. Such system helps to get the real time information regarding traffic conditions in road sections of interest; therefore all this information can be efficiently used to control the transport traffic. All signals from digital video cameras are transmitted to central systems that analyze traffic flows. Presently the statistical information is collected in the real operating objects where video cameras with software support are connected that are able to recognize cars and their licence numbers. When this information is collected it is possible to evaluate efficiency of information collecting subsystem in detail [2-8].

\section{Research on ITS technologies}

\subsection{Functions of ITS components}

Intelligent transport systems are a product of the revolution in information and communications technologies that is the hallmark of the digital age [9]. ITS now support the operation of integrated transport networks, the control of vehicles operating on the networks, and the efficient planning of operations which use those vehicles (including individual journey planning and fleet logistics). They include a wide range of user support functions, from simple information alerts through to highly sophisticated control systems [10].

Essentially, these ITS services can be thought of as an information chain, as shown in Figure 1. The information chain includes data acquisition (from the transportation system), communications, data processing, information distribution, and information utilisation (for decision and control support for the ITS users.) Note that certain external factors like weather forecasting also enter the information chain [4-7]. 


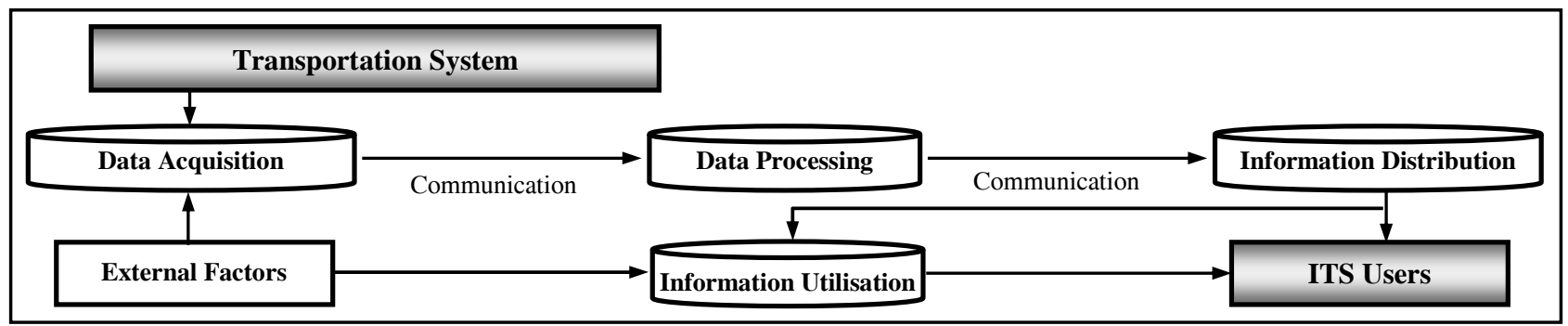

Fig 1. ITS information chain

The concept of an information chain is not new to those who have managed comprehensive traffic systems. As relatively new in ITS, however, are the technologies and system concepts for:

- information exchange and decision coordination involving multiple centres (such as between traffic and transit management centres for intermodal transportation services)

- information acquisition and integration between the vehicle and the road infrastructure (for such functions as dynamic route guidance)

- information exchange with new private-sector organisations (e.g. for information service providers to distribute traffic information through cellular phones or the Internet)

- information exchange with non-transport organisations (e.g. in electronic payment systems involving financial institutions, and in border crossings involving customs and immigration agencies)

\subsection{Enabling technologies}

Table 1 shows examples of enabling technologies for ITS and a way of categorising them. The rows represent the sub-functions of the information chain discussed above. The two right-hand columns segregate the technologies into the infrastructure side and the vehicle side. This demarcation is to facilitate discussion and should not be taken literally since some of the technologies require intimate interaction between the two sides in order to function effectively (such as in electronic toll collection). In addition, technologies used by the traveller (e.g. a cell phone displaying traffic information) can be on either side, depending on whether the traveler is using it at home or in a vehicle.

Table 1. ITS enabling technologies

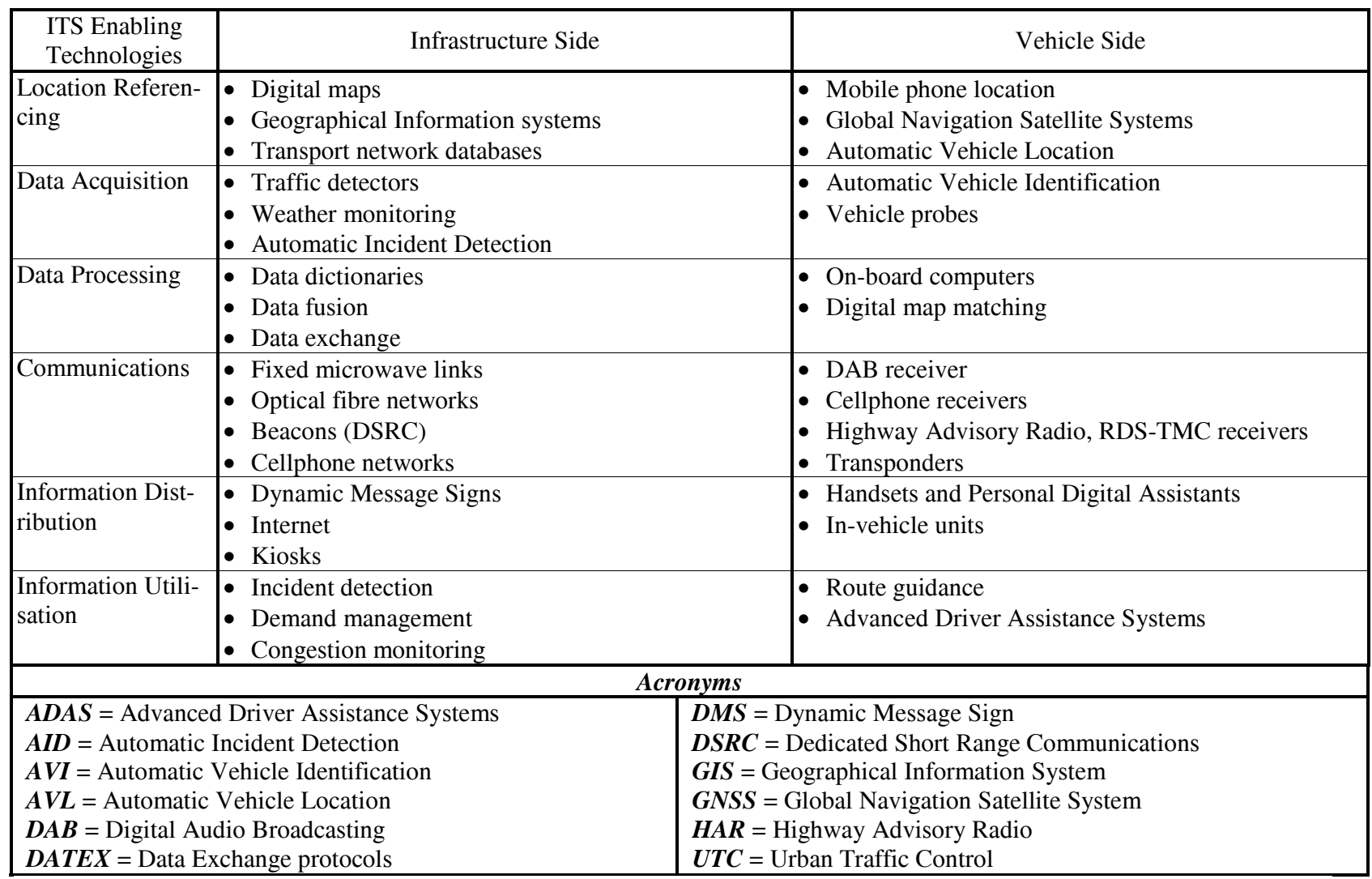


Note that some of these technologies, such as Highway Advisory Radio and UTC, are already familiar to many transportation professionals. Others may be relatively new, including those transferred from the defence industry in the past decade, such as GNSS and the Internet. However, all of these technologies are available off the shelf in the open market [10].

\section{Data Acquisition}

\subsection{Road-based data sources}

A prerequisite for many ITS services is the collection of timely, accurate and reliable information about traffic flow and road conditions. Traffic data falls into three classes: point traffic stream data (e.g. average speed); individual vehicle data (e.g. vehicle type); and link traffic data (e.g. average travel time).

For many years, traffic surveillance has been achieved by inductive loop detectors, which can sense the presence of a vehicle. A single loop buried under the lane pavement can perform vehicle counting. Double loops in the same lane separated by a fixed distance can measure vehicle speed. As vehicle speed slows below a certain threshold, loop detectors can indicate traffic congestion.

Other types of traffic sensors, e.g. ultrasonic, radar, and infrared traffic sensors, are installed on overhead gantries, making their installation and maintenance less disturbing to traffic flow than loop detectors. However, these sensors may not be as reliable as inductive loops in bad weather conditions. In addition, like loop detectors, these sensors work only as single-zone traffic detectors.

Video image detectors (VID) through image processing are one of the more recent technologies to be applied to traffic detection. Images acquired by video cameras in VID are processed to obtain vehicle presence, speed, lane occupancy, lane flow rate, etc. Multiple detection zones can be defined within the field of view of the video camera, thus providing multiple lane coverage by a single camera. Multiple cameras can be connected to one processor unit providing wide area coverage and, coupled with computer software, can reduce the problems caused by shadows, occlusion, and direct sunlight shining on the cameras.

While traffic sensors can provide many attributes of traffic flow, directly or indirectly, there is nothing better than live video images to help the traffic centre operator monitor complicated traffic situations and make appropriate decisions. Visual images from closed circuit television (CCTV) are therefore obtained by the traffic management centre to complement the traffic detectors. Even with a combination of traffic detectors and video traffic surveillance, additional inputs from police patrols, helicopter reporters, road maintenance departments, the meteorological office, taxi fleets and increasingly many cell phone call-ins from drivers on the road, are used for traffic information and management. For example, additional inputs can come from sensors measuring the freezing point of the road surfa- ce, enabling network mangers to calculate the required amount of de-icing chemicals, and resulting in road safety improvement as well as substantial cost savings.

On the vehicle side, data regarding vehicle conditions such as speed, fuel level, oil pressure, engine temperature, etc. are familiar to all drivers. Acquisition of these data through in-vehicle sensors is important for vehicle operation and maintenance. From the perspective of road maintenance, surveillance of vehicle weight is also important. It is estimated that one overloaded truck axle causes more road damage than half a million cars. In recent years, surveillance of commercial vehicle weights through weigh-in-motion (WIM), an ITS technology based on load cell, bending plate, piezoelectric or similar principles designed to catch over-weight trucks without requiring all trucks to stop, has produced substantial time saving benefits to both truck drivers and road authorities in many countries.

Measurement of vehicle dimensions by ITS technologies is also needed for some functions in traffic management. For example, over-height detectors (based on the cutting beam principle) can warn the drivers as they approach a tunnel. The combination of automatic vehicle identification and classification is needed for electronic toll collection [10].

\subsection{Vehicle-based data sources}

In ITS, information about vehicle location is important for both the individual driver who wants to know where he or she is in order to navigate or to obtain location-relevant information, and for the fleet operator who wants to track vehicles for fleet management purposes. Vehicle location is invaluable for public agencies to locate a vehicle in trouble for rescue purposes, or to find stolen vehicles or vehicles transporting hazardous materials. Moreover, when the locations of a moving vehicle are known on a link at two different times, the travel time on the link (or link time) can be measured directly. A vehicle used for this purpose is known as a "probe vehicle" or a "floating vehicle", and the corresponding technology, "vehicle probes", is described below. In addition, tyre slippage on an icy road and moisture on the windshield can also be detected and reported by the vehicle along with its location to the traffic centre. In this case, the vehicle serves as a "probe" for both traffic and road/weather conditions. In South Korea, a national automatic vehicle number identification system is used by the police for traffic law enforcement and vehicle crime prevention.

Automatic Vehicle Identification involves equipment installed on the infrastructure. Vehicle probes based on AVI require installation of appropriate equipment on the infrastructure (either roadside beacons where passing vehicles are equipped with a tag or transponder that can be recognised or camera-based licence plate readers which rely on image processing techniques). The same function may be performed by cooperative probe vehicles providing travel time directly to the traffic centre through wireless communications. The concept of vehicle probe technology can also 
be applied without equipment on the road side, and thus applicable anywhere in the world, by using Automatic Vehicle Location (AVL) based on global navigation satellite systems (GNSS).

Functionally, the use of probe vehicles to measure travel time has three challenges: 1) latency: vehicles need time to reach point B before point A-to-point B travel time can be measured; 2) "leakage": some vehicles never reach point $B$; and 3) sample size: too few vehicles travelling on a link. Thus, reliable measurement of travel time by probe vehicles depends on the sample size and successful matching. These issues are being solved, as more vehicle fleets are equipped with AVI and AVL [10].

\subsection{Automatic vehicle identification (AVI) and floating vehicle data (FVD)}

A coded radio signal transmitted from the vehicle passing under the AVI terminal (or beacon) installed on the gantry at a known location can indicate the location of the vehicle at a particular time. The detection of the same vehicle after, at another AVI terminal, can provide the actual travel time in a road network to the traffic centre. An alternative approach is to use licence plate readers for vehicle identification, in which character recognition technology is used to read the plate number from the image captured by cameras installed on the infrastructure. AVI has also been used for enforcing traffic laws by providing reliable surveillance of individual vehicles when they run through red lights, violate security checks, or fail to pay tolls.

Floating vehicle data (FVD). Vehicle probes are becoming more important, because public agencies realise the comparatively higher costs of conventional traffic sensors on the road infrastructure for widespread ITS applications. A good example is the floating vehicle data (FVD) system used by the ITS company for real-time, traffic data collection in the UK and on the trans-European highway network using truck fleets, and long-distance coaches equipped with AVL. With over 30,000 probe vehicles ITS operate the world's largest FVD network.

\subsection{Transport network data sources}

In addition to the data from traffic and vehicles, a considerable investment is needed in data concerning the transport networks themselves. The basis for ITS in many cases is the accumulation of detailed and reliable databases of network links, inter-connections and other features, supported by a sound location referencing system.

Capturing data on transport networks is very labour-intensive, involving detailed reference to maps and plans, aerial photographs, and on-site surveys. Videoing the network from a moving vehicle is often used to reduce-the amount of time spent on the ground. Viewing these video images is an effective form of desk-based data capture. The images can be studied frame-by-frame if detail is required, whereas the fast forward control allows unimportant sections to be skip- ped. At the time of data capturing careful attention must be given to the way the database will be used, since at some future occasion someone will have to interpret the data. Numerical codes, grid references, and latitude and longitude coordinates do not convey any meaning in themselves. Network features need to be described in terms which the user will easily comprehend, including local place names, landmarks, and other descriptions.

With the advent of hand-held GNSS receivers and vehicles equipped with AVL the business of accurately locating network features like intersections, freeway merge and diverge points, bridges, tunnels, access points to properties, transit stops, etc. has become much easier. Without an inventory of stop locations, for example, it is not possible to offer point-to-point journey planning for public transport. Similarly to road information, reliable coding of the network is needed for emergency response, incident reporting and other location based services. The degree of precision in location referencing is especially important. Nothing is worse than having mis-located a feature in the event of an emergency, for example an error which puts the vehicle on the wrong carriageway!

Opportunities exist for automating data capture and eliminating human error. However, the process remains very time-consuming. Transport network databases, like the transport networks themselves, need constant maintenance to keep them up-to-date. Careful checking is essential to avoid errors which can lead to features being incorrectly located, sometimes wildly misplaced. The long-established maxim of "RIRO" (rubbish in, rubbish out) applies to ITS as much as to any other Branch of Information Technology.

\section{Gaining intelligence: data processing}

\subsection{Traffic and travel information}

Traffic information can be obtained in many ways and from many sources at the same time. Thus, at the traffic or transportation management centre, there is a need to process the data, verify their accuracy, reconcile conflicting information, put them into compatible formats, and combine them with data from other agencies (e.g. transit management centre, highway maintenance organisation, police department, etc.). This process is known as data fusion.

One of the important data processing applications is to provide the user service of current traffic information and/or predicted travel times (PTT). This involves the fusion of travel information from relevant sources, both public and private, as in the case of Trafficmaster. For predictive information, data fusion would also include time variability depending on time of departure, roadworks, weather, events, incident reports, etc. Additional factors taken into account in PTT could include vehicle types (car, bus, truck), driver types (neutral, passive, aggressive) as well as specific time (current, or any time within the next 48 hours) [10]. A variety of approaches to PTT can be found in the literature, ranging from the use of analytical algorithms, artificial neural network, to traffic simulation, or a selective 
combination of these methods. The predicted travel time may be displayed not only on Dynamic Message Signs (DMS), but also through the media, In-Vehicle Units (IVU), or handheld devices. Access to PTT through the latter would help travellers make decisions on departure time and/or mode choice (between driving and public transit) in pre-trip planning.

\subsection{Automatic incident detection (AID)}

Automatic incident detection (AID) has proven helpful in reducing incident detection time, and in speeding up rescue operations and traffic diversion around incident sites. There are also other applications of AID. For example, AID has been used to estimate traffic conditions or degree of congestion (slow-moving, congested, free-flow). AID cameras have been used for travel time estimation, based on the idea that AID can determine the mean speed of each segment along the covered stretch. The method is also used to automatically display driver alerts on DMS and to trigger a mandatory speed limit upstream of the incident.

Automatic incident detection is another important data processing technology on the infrastructure side. This is accomplished through computer processing, based on sophisticated algorithms applied to traffic data obtained from a variety of detectors. To determine whether an incident has occurred, the input data from the detection system are tested against an algorithm. The algorithms that have been developed include a number of methodologies such as comparative, statistical forecasting of traffic behaviour, and others, where loop occupancy for more than a set interval indicate stationary or slow-moving vehicles. In general, AID technology is not designed to replace the traffic centre operator but to alert him or her to traffic patterns resembling those in an incident. Human confirmation through CCTV or site visits is still needed. The use of portable AID and CCTV equipment around highway work zones and other temporary high risk locations is especially valuable.

Positive confirmation of the end of an incident is as important as early detection. It is essential to identify reliably the beginning and the end of an incident so that drivers are not given wrong advice which destroys confidence in the ITS systems. DMS alerts need to be displayed quickly and cancelled promptly when not needed. Similarly, temporary speed limits should be imposed as soon as a dangerous incident arises, and should be lifted as soon as the road is clear. Spot speed detection and point-to-point journey time measurements using probe vehicles are useful ways to recognise that everything is back to normal, as well as remote observation of traffic conditions using CCTV.

\subsection{Vehicle location and navigation}

Since all satellite navigation systems require the observation of at least four satellites to function, vehicle location needs complementary systems that still work while the vehicle is in a tunnel, under trees, or in an "urban canyon" surrounded by tall buildings. These gaps in coverage can be bridged with map matching, the basic component of popular in-vehicle navigation systems. These take advantage of the fad that vehicle location is usually restricted to the road network except during temporary deviations when the vehicle is in a parking lot or on a ferry. As the name implies, map matching uses a highly accurate digital map on the vehicle and heuristic algorithms to deduce where the vehicle is located on the map. Another approach to navigation is dead reckoning, which uses a gyroscope or related inertial guidance principles to deduce vehicle location in reference to a known starting point. However, dead reckoning cannot function alone since the cumulative error needs to be corrected from time to time, preferably automatically.

Digital maps are a prerequisite for a number of advanced traveller information and route guidance systems. To create digital maps, raw road network data is collected from digitised and paper maps, aerial photographs, and other information sources. The information is combined with navigation-enabling attributes, and then digitised with the aid of specialised computer software.

This data is compiled by the navigation hardware suppliers and there is a major ongoing effort to keep the data updated with the ever changing road network. Transport networks are evolving all the time. Some changes are permanent, like the opening of a new length of highway. Others, like the closure of a strategic link for bridge repairs, are temporary. Users can purchase or subscribe to regular map updates on CD-ROM, but some vehicles are equipped to "learn" road links which may be missing from the installed version of the map database. Another way is to broadcast the latest map updates over a digital data channel, so that even temporary road closures can be logged and stored.

With advanced data compression and storage technology, a digital map showing all the major roads in the United States can now be stored in a single compact disk (DVD). For information needed for local route guidance, the digital map of a metropolitan area may be put in a PCM-CIA card (also known as a PC card). Digital maps are also key enablers of location-aware and location-based services, used in traffic information and incident management, which rely on unambiguous vehicle position and location of traffic congestion. As noted above, the need and cost of updating digital maps should not be underestimated. The development of the Terra-S Road Database in Slovakia shows how the private and public sectors have collaborated to provide a comprehensive geospatial road database essential for the development of ITS.

There are other methods for determining vehicle location, especially using cell phones. Such methods can be important for emergency calls from mobile phones (extended 911 or E911 service in the USA) with automatic indication to the rescue team where the caller is located, as well as to enable a host of locationspecific ITS services. 


\subsection{Location-based Services}

Among the host of potential and available location-based services, many customers (end users) put their highest priorities on safety and security, which has led to the provision of rescue services as one of the earliest ITS offerings in the market place. Distress signals may be conveyed to the rescue centre manually or automatically, e.g. triggered by airbag deployment, and the location of the vehicle in distress is conveyed automatically and precisely by GNSS. Such rescue services are often made available in combination with other ITS services such as stolen vehicle tracking and driving directions.

\section{ITS data acquisition technologies}

In order to select optimal ITS structure, subsystems, and location points it is important to evaluate efficiency of these systems. General (overall) ITS system efficiency can be separated into two parts: 1. efficiency of information acquisition and transmission and degree by which this information satisfies needs; 2. control quality: parameters of control extent, adaptability, and partial maintenance duration reduction of transport means. Degree by which information required by ITS satisfies needs can be evaluated using data acquisition systems of different technologies [1, 2].

General ITS data acquisition technology adequacy for gathering required information will be as follows:

$$
Q_{\text {Techn }}=\frac{\sum_{i=1}^{n} q_{i j} \cdot \rho_{i}}{n},
$$

where: $j$ - number of data acquisition technology, $i-$ number of information type, $q_{i j}$ - normalized data acquisition adequacy index of $j$-technology for gathering of particular $i$-information, $\rho_{i}-$ normalized significance coefficient of information of $i$-type [3].

After performing expert survey the data have been received which evaluate the adequacy of currently used technologies for gathering required information. The data are presented in Table 2.

\section{ITS efficiency}

Efficiency of overall Intelligent Transportation System, which uses video cameras to gather information, depends on the following parameters: efficiency of video cameras $E_{K}$; efficiency of placement of video cameras $E_{I}$; system of data acquisition via video cameras operation efficiency $E_{S}$; efficiency of information transmission networks $E_{T}$; efficiency of control algorithm $E_{A}$.

Efficiency of video cameras. Some of main features of video cameras are directional characteristic, resolution, sensitivity to lighting, focal length of lenses, etc 3]. All these features are characterized by the set $\left\{L_{j}\right\}$ of indexes $\left(j=1, L_{J}\right)$, values of these indexes are expressed by the set $\left\{L_{j}\right\}$. Thus technical level of video camera according to all technical indexes can be expressed using equation:

$$
Q_{K}=\sqrt{\sum_{j=1}^{L_{j}}\left(q_{j} \cdot \eta_{j}\right)^{2}},
$$

where: $j$ - technical index of video camera; $q_{j}-$ normalized value of parameter of $j$-index; $\eta_{j}-$ significance coefficient of $j$-index [3].

Not all parameters of video cameras used to recognize transport means are equally significant. A grade is attributed to more important indexes:

$$
g_{j / u=1}=m+1
$$

and for not so important

$$
g_{j / u=2,3, \ldots m}=m-u+1,
$$

where: $u$ - number of $j$-index. Therefore significance coefficient expression will be:

$$
\eta_{j}=\frac{g_{j}}{\sum_{i=1}^{m} g_{i}}
$$

Table 2. Evaluation of technology adequacy for gathering required information

\begin{tabular}{|l|c|c|c|c|c|}
\hline Information & $\begin{array}{c}\text { Video image } \\
\text { detection }\end{array}$ & $\begin{array}{c}\text { Technologies of } \\
\text { contact data } \\
\text { gathering }\end{array}$ & Microwave radar & $\begin{array}{c}\text { Vehicle marking } \\
\text { tags }\end{array}$ & Etc. \\
\hline Climate conditions & $q_{11}$ & $\ldots \ldots$ & $q_{1 j}$ & $\ldots \ldots$ & $q_{1 m}$ \\
\hline Length of standing cars queue & $q_{21}$ & $\ldots \ldots$ & $q_{2 j}$ & $\ldots \ldots$ & $q_{2 m}$ \\
\hline Vehicle classification & $\ldots \ldots$ & $\ldots \ldots$ & $\ldots \ldots$ & $\ldots \ldots$ & $\ldots \ldots$ \\
\hline Occupation on road & $q_{i 1}$ & $\ldots \ldots$ & $q_{i j}$ & $\ldots \ldots$ & $q_{i m}$ \\
\hline Accidents & $\ldots \ldots$ & $\ldots \ldots$ & $\ldots \ldots$ & $\ldots \ldots$ \\
\hline Vehicle identification & $\ldots \ldots$ & $\ldots \ldots$ & $\ldots \ldots$ & $\ldots \ldots$ & $\ldots \ldots$ \\
\hline Etc. & $q_{1 n}$ & $\ldots \ldots$ & $q_{n j}$ & $\ldots \ldots$ & $q_{n m}$ \\
\hline
\end{tabular}


Not only image indexes are important for ITS systems, but also expenses of video camera installation [8]. It is necessary to establish a graph of each camera type quality coefficient dependency on cost in order to select most suitable video cameras, on one axis of which technical level coefficient $\left(Q_{K i}\right)$ is represented, and the value of economic index (cost) $\left(C_{K i}\right)$ for this camera type is represented on the other axis (Fig 2).

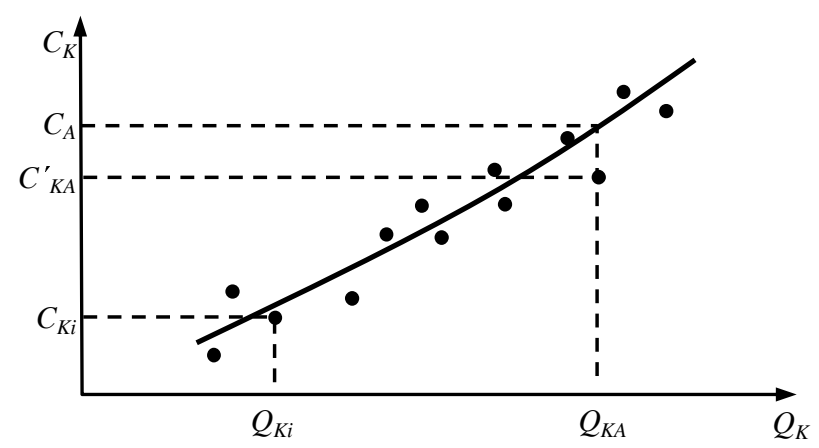

Fig 2. Relation between technical level and price

This curve can be used for evaluation of general (overall) efficiency of video cameras. Consequently selection efficiency of particular video camera [6] is expressed as:

$$
E_{K A}=\left\{\begin{array}{l}
1-\frac{C_{K A}^{\prime}-C_{K A}}{C_{K A}} K_{T}, \text { when } C_{K A}^{\prime}>C_{K A} ; \\
1, \text { when } C_{K A}^{\prime} \leq C_{K A} .
\end{array}\right.
$$

\section{Conclusions}

1. Intelligent Transport Systems work with information and control technologies which provide the core of ITS functions [9]. Some of these technologies, like loop detectors, are well known to transportation professionals. However, there are a number of less familiar technologies and system concepts that are key to ITS functions.

2. Essentially, these ITS services can be thought of as an information chain, as shown in Fig 1. The information chain includes data acquisition (from the transportation system), communications, data processing, information distribution, and information utilisation (for decision and control support for the ITS users).

3. General ITS system efficiency can be separated into two parts: 1) efficiency of information acquisition and transmission and degree by which this information satisfies needs; 2) control quality: parameters of control extent, adaptability, and partial maintenance duration reduction of transport means.

4. General ITS data acquisition technology adequacy for gathering required information is defined by Formula (1).

\section{References}

1. RAN, B. Using Traffic prediction models for providing predictive traveler information. International Journal of Technology Management (special issue on the impact of information systems technology on operations management), 2004, p. 12-19.

2. HUDDART, K. W. Traffic control. In J. Walker (ed.). Advances in Mobile Information Systems. Boston: Artech House, 2003, p. 140-153.

3. KRAMER, U. (ed.); PRESTON, N. (eds.). Manmachine interface-black bool. Report to the PROMETHEUS Steering Commite, PRO-CAR 1, Prometheus Office, Stutgard, 2000.

4. Telaid design guidelines handbook: for usability of invehicle systems by drivers with special needs http://hermes.civil.auth.gr/guide.html (Aristotle University of Thessaloniki).

5. SHERRY, L. Report on non-traditional traffic country methods. Tanque Verde Loop Rd. Tucson, AZ 85748, 2001.505 p.

6. OAKLAND, S.; FOLLOWER, R. Statistical process control, fifth edition. Cornwall: MPG Books Limited, 2003. $460 \mathrm{p}$.

7. KEITH, J. Video Demystified: a handbook for the digital engineering, third edition. Eagle Rock: LLH Technology Publishing, 2001. 782 p.

8. BALAISIS, P.; EIDUKAS, D.; VALINEVICIUS, A.; ZILYS, M. Information electronic systems efficiency. Kaunas: Technologija, 2004.

9. JARAŠŨNIENE, A. Analysis of possibilities and proposals of intelligent transport systems (ITS) implementation in Lithuania. Transport, 2006, Vol XXI, No 4, 245-252.

10. MILES, C. J.; CHEN, K. The intelligent transport system. Handbook. UK, 2004, p. 34-48. 